\title{
Tamanho de partícula do volumoso e freqüência de alimentação sobre o metabolismo energético e protéico em ovinos, considerando dietas com elevada participação de concentrado ${ }^{1}$
}

\author{
Forage particle size and the number of feedings over energy and protein metabolism in \\ sheep
}

\author{
GOMES, Silas Prímola²; BORGES, Iran ${ }^{3 *}$; BORGES, Ana Luíza Costa Cruz³ ; \\ MACEDO JUNIOR, Gilberto de Lima ${ }^{4}$; CAMPOS, Warley Efren ${ }^{5}$; BRITO, Túlio \\ Soares de ${ }^{3}$
}

\footnotetext{
${ }^{1}$ Parte da tese de doutorado do segundo autor. Trabalho financiado com recursos do CNPq e rações Itambé.

${ }^{2}$ Agroceres, São Paulo, São Paulo, Brasil.

${ }^{3}$ Universidade Federal de Minas Gerais, Escola de Veterinária, Departamento de Zootecnia, Belo Horizonte, Minas Gerais, Brasil.

${ }^{4}$ Universidade Federal de Uberlândia, Faculdade de Medicina Veterinária e Zootecnia, Uberlândia, Minas Gerais, Brasil.

${ }^{5}$ Universidade Federal de Minas Gerais, Escola de Veterinária, Programa de Pós-Graduação em Ciência Animal, Belo Horizonte, Minas Gerais, Brasil.

*Endereço para correspondência: gilbertomacedojr@gmail.com
}

\section{RESUMO}

Objetivo-se avaliar o efeito diferente de tamanhos de partículas do volumoso e a frequência de alimentação sobre o metabolismo dos animais. Oito carneiros com peso vivo médio de $52,5 \mathrm{~kg}$ foram distribuídos em esquema fatorial $2 \times 4$, de modo a corresponder a duas frequências de alimentação e quatro tamanhos (2mm; 5mm; $10 \mathrm{~mm}$ e $25 \mathrm{~mm})$, em delineamento em blocos ao acaso. Foi utilizado o feno de Tifton-85 (Cynodon spp.) como volumoso e concentrado comercial, o que impôs um ajuste na relação volumoso:concentrado, que assim ficou quantificada: $25: 75 \%$. O trabalho de respirometria foi conduzido em câmara respirométrica, tipo circuito aberto, para pequenos ruminantes. Foram determinados os teores de oxigênio, gás carbônico e metano. $\mathrm{Na}$ urina, foi determinado o teor de nitrogênio. A partir desses parâmetros, foi determinada a produção de calor (PC). O nitrogênio retido representou $36,0 \%$ do Nitrogênio $(\mathrm{N})$ ingerido (g/dia) e $46,8 \%$ do $\mathrm{N}$ absorvido (g/dia). A produção média de $\mathrm{CH} 4$ foi de 1,45L/UTM/dia. A PC foi cerca de $40,0 \%$ da energia bruta ingerida. A energia metabolizável foi $73,2 \%$ da Energia bruta ingerida e a energia líquida apresentou valor médio de $1.807,35 \mathrm{kcal} / \mathrm{dia}$.
Não houve efeito dos tamanhos de partícula e da frequência sobre o balanço de nitrogênio e o metabolismo energético.

Palavras-chave: calorimetria, energia líquida, fibra fisicamente efetiva, respirometria.

\section{SUMMARY}

This study aimed to evaluate the effect of different forage particle sizes and the feeding frequency on the animals metabolism. Eight lambs with $52.5 \mathrm{~kg}$ live weight were distributed in a randomized block design according the $2 \times 4$ factorial, corresponding to two feeding frequencies and four particle sizes $(2 \mathrm{~mm} ; 5 \mathrm{~mm}$; $10 \mathrm{~mm}$ and $25 \mathrm{~mm})$. The forage used was the Tifton-85 hay (Cynodon spp.) and commercial concentrate, adjusting the forage: concentrate ratio to $25: 75 \%$. The respirometry experiment was conducted in an open circuit respiration chamber for small ruminants. The concentrations of oxygen, carbon dioxide and methane were determined. Nitrogen content was determined in urine. With these parameters was determined heat production (PC). The nitrogen retained represented $36.0 \%$ of Nitrogen $(\mathrm{N})$ 
intake (g/day) and $46.8 \%$ of $\mathrm{N}$ absorbed (g/day). The $\mathrm{CH} 4$ average production was $1.45 \mathrm{~L} / \mathrm{UTM} /$ day. The PC was approximately $40.0 \%$ of crude energy intake. The metabolizable energy was $73.2 \%$ of crude energy intake and the average value of net energy was $1807.35 \mathrm{kcal} /$ day. There was no effect of particle size and feeding frequency on the nitrogen balance and energy metabolism.

Keywords: calorimetry, net energy, physically effective fiber, respirometry.

\section{INTRODUÇÃO}

A adoção da energia líquida (EL) como forma de expressar as exigências dos animais e o conteúdo energético dos alimentos pode tornar mais precisa a formulação de rações para os ruminantes.

A energia retida nos tecidos corporais ou utilizada na produção é calculada como a diferença entre a energia bruta (EB) total consumida e as perdas de energia nas fezes, na urina, nos gases produzidos e na produção de calor (BLAXTER, 1956). Nos ruminantes, uma das principais formas de perda de energia ocorre através da liberação de $\mathrm{CH}_{4}$, que pode variar de $5-8 \%$ da energia bruta ingerida (NRC, 2007). A perda de energia, na forma de metano $\left(\mathrm{CH}_{4}\right)$, aumenta com o incremento de forragem na dieta (VAN SOEST, 1994) e é afetada também pelo nível de consumo e pelo uso de aditivos.

Outro fator que pode influenciar a produção de $\mathrm{CH}_{4}$ é o processamento dos alimentos e o manejo nutricional que podem afetar o consumo, a taxa de passagem e a digestibilidade ruminal e intestinal dos volumosos e concentrados. $\mathrm{O}$ consumo e os coeficientes de digestibilidade aparente dos nutrientes são influenciados por fatores como o teor de nutrientes, pelos efeitos associativos entre os alimentos, pela relação
Volumoso:Concentrado e pelo processamento dos alimentos (ALVES et al., 2003; FURUSHO-GARCIA et al., 2003; SILVA et al., 2005; MACEDO JUNIOR et al., 2006; SILVA et al., 2007; ARRUDA et al., 2008; ZANINE \& MACEDO JUNIOR, 2006; MACEDO JUNIOR et al., 2009; GOMES et al., 2012). O tempo de ruminação é, consideravelmente, influenciado pelas características físicas da dieta, como tamanho de partícula (FRANÇA et al., 2009), e pelo manejo nutricional adotado, como número de refeições (DOREAU \& DOREAU, 2001; DEVRIES et al., 2005; DOREAU, et al., 2003). A determinação da produção de calor (PC) pelo animal em jejum é importante, pois representa a energia gasta com a mantença (RODRIGUEZ et al., 2007). A determinação do teor de nitrogênio $(\mathrm{N})$ retido nos tecidos corporais é uma importante forma de avaliação do valor nutricional das dietas para ruminantes. Quanto maior for a degradabilidade da proteína da ração, maior será a produção de amônia e, possivelmente, maiores serão as perdas urinárias de compostos nitrogenados na forma

de ureia (DOREAU et al., 2003). Assim, foram avaliados os efeitos do tamanho de partícula do volumoso e de dois manejos alimentares sobre o metabolismo energético, o valor energético da dieta e o balanço de nitrogênio em ovinos.

\section{MATERIAL E MÉTODOS}

O experimento foi realizado no Laboritório de Calometria e Respirometria Animal, localizado na Escola de Veterinária da UFMG em Belo Horizonte (MG) nos meses de outubro, novembro e dezembro de 2005. As análises 
laboratoriais foram realizadas nas dependências do Laboratório de Nutrição do Departamento de Zootecnia da EVUFMG. As temperaturas máximas e mínimas foram, respectivamente, de $31^{\circ} \mathrm{C}$ e $21^{\circ} \mathrm{C}$ e os valores máximos e mínimos de umidade relativa do ar foram, respectivamente, de $90,0 \%$ e $28,0 \%$, durante o período experimental.

Foram utilizados oito carneiros, com peso vivo médio de $52,5 \mathrm{~kg}$, alojados em gaiolas de metabolismo, com o cocho separação para concentrado e volumoso. Foi empregado o feno de Tifton-85 (Cynodon spp.) como volumoso e um concentrado comercial de manutenção constituído de milho moído (17,2\%), farelo de glúten de milho $(8,3 \%)$, gérmen de milho $(15,0 \%)$, casca de soja $(24,0 \%)$, resíduo de feijão $(18,0 \%)$, farelo de babaçu $(12,0 \%)$, óleo vegetal
$(1,0 \%)$, melaço líquido $(1,0 \%)$, cloreto de sódio $(0,6 \%)$, calcário calcítico $(2,8 \%)$ e premix mineral vitamínico $(0,1 \%)$. As dietas foram balanceadas de acordo com o NRC (1985) para ganho de peso: as sobras foram quantificadas em 5\% e, na relação $\mathrm{V}: \mathrm{C}$, quantificou-se 25:75\%. A escolha dessa relação volumoso:concentrado objetivava testar a capacidade dos animais em trabalhar com dietas, compostas por alto teor de concentrado. A fim de verificar a condição metabólica dos animais, utilizou-se o volumoso em 4 tamanhos diferentes (Tabela 2) e com dois manejos de alimentação (duas e quatro refeições ao dia). A composição bromatológica do volumoso, do concentrado e da dieta experimental encontra-se na Tabela 1.

Tabela 1. Composição bromatológica do feno, do concentrado e da dieta utilizada

\begin{tabular}{lrrr}
\hline Item & Feno & Dieta $^{1}$ & Concentrado \\
\hline Matéria seca (\%) & 84,78 & 85,28 & 85,45 \\
Matéria orgânica & 94,91 & 91,88 & 90,87 \\
Proteína bruta & 10,33 & 17,03 & 19,27 \\
Extrato etéreo & 2,31 & 3,02 & 3,25 \\
Cinzas & 5,09 & 8,12 & 9,13 \\
Carboidratos não fibrosos & 4,05 & 21,59 & 27,43 \\
Fibra em detergente neutro & 78,22 & 50,24 & 40,92 \\
Fibra em detergente ácido & 35,90 & 26,37 & 23,20 \\
Lignina & 3,05 & 3,85 & 4,12 \\
\hline${ }^{1}$ Dieta contendo 75\% de concentrado. & & &
\end{tabular}

${ }^{\mathrm{I}}$ Dieta contendo $75 \%$ de concentrado.

Foram adotados dois manejos alimentares com duas refeições diárias, às $7 \mathrm{~h}$ e $19 \mathrm{~h}$ (tratamento F2), denominados de F2 e F4, respectivamente: duas refeições diárias às $7 \mathrm{~h}$ e $19 \mathrm{~h}$ ou quatro refeições diárias às $7 \mathrm{~h}$; $11 \mathrm{~h} ; 15 \mathrm{~h}$ e $19 \mathrm{~h}$. Diferentes tamanhos de partícula do feno foram avaliados a partir da desintegração do feno e separados nos tamanhos de $2 \mathrm{~mm} ; 5 \mathrm{~mm} ; 10 \mathrm{~mm}$ e $25 \mathrm{~mm}$; que constituíam, aproximadamente, $500 \mathrm{~g}$ do material obtido em cada peneira.
Foram colocados no separador de partícula modelo Penn State, que continham duas peneiras (19 e $8 \mathrm{~mm}$ de porosidade) e uma bandeja de fundo. $\mathrm{O}$ estudo das partículas e seu diferentes tamanhos foi baseado nas considerações de Lammers et al. (1996). O perfil de distribuição (\%) da fibra em detergente neutro (FDN) das partículas do feno e os diâmetros geométricos médios (Dgm) dos 
diferentes tratamentos são apresentados na Tabela 2.

$\mathrm{O}$ fator de efetividade física (fef) dos diferentes tamanhos de partícula foi determinado como a fração da MS retida acima da peneira de $8 \mathrm{~mm}$ de porosidade, e a fibra em detergente neutro fisicamente efetiva (FDNfe), obtida pela multiplicação do fef pelo teor da FDN da amostra de feno. O Dgm e o desvio-padrão geométrico médio (Sgm) foram calculados conforme o ASAE (2001), citado por Kononoff \& Heinrichs (2003).

Foi realizado ensaio de digestibilidade aparente em quatro períodos experimentais e realizou-e a adaptação às dietas durante 14 dias. Foram pesados e amostrados, diariamente, os alimentos oferecidos, as sobras e as fezes. Fez-se um pool das amostras de cada animal, dos cinco dias coletados. As fezes foram recolhidas em bandejas plásticas e a urina acondicionada em baldes adaptados, com tela separadora, no qual havia $100 \mathrm{~mL}$ de ácido sulfúrico $2 \mathrm{~N}$. As amostras dos alimentos, das sobras e das fezes foram pré-secas em estufa ventilada, regulada a $55^{\circ} \mathrm{C}$, moídas a $1 \mathrm{~mm}$, e a urina mantida congelada. Para as amostras de alimentos oferecidos, sobras e fezes foram realizadas as análises de MS a $105^{\circ} \mathrm{C}$ e proteína bruta (PB), e na urina a análise de $\mathrm{PB}$, conforme técnicas descritas por Silva \& Queiroz (2002). As análises de FDN foram realizadas de acordo com os métodos de Van Soest et al. (1991).

Tabela 2. Perfil de distribuição (\%) da matéria seca (MS) e da fibra em detergente neutro (FDN) das partículas dos tratamentos

\begin{tabular}{lrrrr}
\hline \multirow{2}{*}{ Tamanho de partícula } & \multicolumn{4}{c}{ Tratamento } \\
\cline { 2 - 5 } & $2 \mathrm{~mm}$ & $5 \mathrm{~mm}$ & \multicolumn{3}{c}{$10 \mathrm{~mm}$} \\
\cline { 2 - 5 } & \multicolumn{4}{c}{ \% MS retida } \\
\hline$>19 \mathrm{~mm}$ & 0,00 & 6,00 & 65,29 & 82,38 \\
$8-19 \mathrm{~mm}$ & 25,50 & 27,30 & 3,34 & 13,67 \\
$<8 \mathrm{~mm}$ & 74,50 & 72,70 & 31,37 & 3,95 \\
Dgm $(\mathrm{mm})$ & 1,65 & 1,74 & 9,45 & 23,05 \\
Sgm $(\mathrm{mm})$ & 3,91 & 4,10 & 18,92 & 30,13 \\
\hline & & \multicolumn{4}{c}{$\%$ FDN retida } \\
\hline$>19 \mathrm{~mm}$ & 0,00 & 0,00 & 66,36 & 82,07 \\
$8-19 \mathrm{~mm}$ & 24,39 & 25,86 & 3,25 & 13,81 \\
$<8 \mathrm{~mm}$ & 75,61 & 74,14 & 30,39 & 4,12 \\
Fef & 0,24 & 0,26 & 0,70 & 0,96 \\
FDNfe $(\% \mathrm{MS})$ & 18,77 & 20,34 & 54,75 & 75,09 \\
\hline
\end{tabular}

TP2 = tamanho de partícula de $2 \mathrm{~mm}$; TP5 = tamanho de partícula de $5 \mathrm{~mm}$; TP10 = tamanho de partícula de $10 \mathrm{~mm}$; TP25 = tamanho de partícula de $25 \mathrm{~mm}$.

Dgm = diâmetro geométrico médio calculado conforme ASAE (2001); Sgm = desvio padrão geométrico médio calculado conforme ASAE (2001). fef $=$ fator de efetividade física $[(100-\%$ de MS < $8 \mathrm{~mm}) / 100]$; FDNfe (\%MS) - fibra em detergente neutro fisicamente efetiva (fef $* \%$ FDN do feno) expressa como porcentagem da matéria seca.

O trabalho de respirometria foi conduzido em câmara respirométrica individual, do tipo circuito aberto, para pequenos ruminantes, com coletas de amostras dos gases realizadas a cada 15 minutos e duração total de 24 horas. Os animais foram previamente adaptados ao ambiente da câmara por 48 horas, 
cuja temperatura mantida era entre $20 \mathrm{e}$ $25^{\circ} \mathrm{C}$. As alíquotas do ar foram retiradas, automaticamente, por bomba e canalizadas até os analisadores para determinação dos teores de $\mathrm{O}_{2}, \mathrm{CO}_{2}$ e $\mathrm{CH}_{4}$. A PC foi determinada por meio da equação de Brouwer (1965): $\mathrm{PC}(\mathrm{kJ})=$ $16,18 \mathrm{O}_{2}+5,02 \mathrm{CO}_{2}-2,17 \mathrm{CH}_{4}-5,99$ NUR. O coeficiente respiratório (RQ) foi calculado pela relação entre o $\mathrm{CO}_{2}$ produzido/ $\mathrm{O}_{2}$ consumido.

$O$ teor de EB dieta oferecida, das sobras, das fezes e da urina foi determinado em bomba calorimétrica, a ED da dieta foi determinada pela diferença entre a $E B$ ingerida e a $E B$ excretada nas fezes. A EM da dieta correspondeu à ED subtraída da energia bruta liberada na produção de metano e de urina, e a EL obtida pela diferença entre a EM e a energia da produção de calor. Foi adotado o valor de energia do $\mathrm{CH}_{4}$ 9,44kcal/L de acordo com Blaxter \& Clapperton (1965). Todos os valores, com exceção do NUR, foram expressos em $\mathrm{kcal} / \mathrm{dia}$ ou $\mathrm{kcal}$ por unidade de tamanho metabólico $\left(\mathrm{UTM}=\mathrm{PV}^{0,75}\right)$. Foi determinado também o balanço de nitrogênio $(\mathrm{N})$, no qual obteve-se o $\mathrm{N}$ retido $(\mathrm{g} / \mathrm{d})$ pela diferença entre $\mathrm{o} N$ consumido (g/d) e o $\mathrm{N}$ excretado nas fezes e urina $(\mathrm{g} / \mathrm{d})$.

Foi adotado esquema fatorial $2 \times 4$, com duas frequências de alimentação e quatro tamanhos de partícula do feno, em delineamento em blocos ao acaso, com quatro repetições (animais). Para comparação das variáveis estudadas, utilizou-se análise de regressão a 5\% de probabilidade, com ajuste dos modelos lineares e quadráticos. A definição do modelo foi feita em cima da probabilidade e da resposta biológica da variável estudada. Algumas respostas apresentaram coeficiente de variação acima de $40 \%$, possivelmente, por fator ligado a própria variável.

\section{RESULTADOS E DISCUSSÃO}

As médias e os coeficientes de variação (CV\%) do balanço de nitrogênio são apresentados na Tabela 3. Os tratamentos (F2 e F4 e 2mm; 5mm; $10 \mathrm{~mm}$ e $25 \mathrm{~mm}$ ), bem como a interação entre eles não afetaram os parâmetros estudados $(\mathrm{P}>0,05)$.

Tabela 3. Médias e coeficientes de variação (CV) do balanço de nitrogênio (N) nos diferentes tratamentos

\begin{tabular}{|c|c|c|c|c|c|c|c|c|c|c|}
\hline \multirow{3}{*}{ Item } & \multicolumn{8}{|c|}{ Tratamentos } & \multirow{3}{*}{ Média } & \multirow{3}{*}{$\begin{array}{l}\text { CV } \\
(\%)\end{array}$} \\
\hline & \multicolumn{4}{|c|}{$\mathrm{F} 2$} & \multicolumn{4}{|c|}{$\mathrm{F} 4$} & & \\
\hline & $2 \mathrm{~mm}$ & $5 \mathrm{~mm}$ & $10 \mathrm{~mm}$ & $25 \mathrm{~mm}$ & $2 \mathrm{~mm}$ & $5 \mathrm{~mm}$ & $10 \mathrm{~mm}$ & $25 \mathrm{~mm}$ & & \\
\hline $\mathrm{N}$ ingerido $(\mathrm{g} / \mathrm{dia})^{*}$ & 29,19 & 36,40 & 27,22 & 33,29 & 39,52 & 36,76 & 36,19 & 32,55 & 33,89 & 24,30 \\
\hline $\mathrm{N}$ fecal (g/dia)* & 7,75 & 8,65 & 5,93 & 7,36 & 8,66 & 8,43 & 8,74 & 7,38 & 7,86 & 33,16 \\
\hline $\mathrm{N}$ absorvido (g/dia)* & 21,43 & 27,74 & 21,28 & 25,92 & 30,85 & 28,33 & 27,44 & 25,16 & 26,02 & 22,94 \\
\hline $\mathrm{N}$ urinário $(\mathrm{g} / \mathrm{dia})^{*}$ & 13,15 & 15,06 & 12,77 & 13,37 & 15,07 & 13,89 & 13,34 & 12,95 & 13,70 & 23,46 \\
\hline $\mathrm{N}$ retido $(\mathrm{g} / \mathrm{dia})^{*}$ & 8,28 & 12,69 & 8,51 & 12,55 & 15,78 & 14,43 & 14,10 & 12,21 & 12,31 & 37,94 \\
\hline $\mathrm{N}$ retido $(\mathrm{g} / \mathrm{UTM}) *$ & 0,47 & 0,67 & 0,42 & 0,63 & 0,84 & 0,67 & 0,75 & 0,63 & 0,63 & 37,75 \\
\hline $\mathrm{N}$ retido/ $\mathrm{N}$ ing. $(\%)^{*}$ & 29,96 & 33,44 & 32,64 & 38,37 & 40,14 & 38,49 & 38,57 & 36,42 & 36,00 & 24,38 \\
\hline $\mathrm{N}$ retido/ $\mathrm{N}$ abs. $(\%)^{*}$ & 38,71 & 45,75 & 39,98 & 48,43 & 51,12 & 50,91 & 51,38 & 48,50 & 46,84 & 22,75 \\
\hline
\end{tabular}


Pode-se observar que o balanço de nitrogênio foi positivo, já que o $\mathrm{N}$ retido representou $36,0 \%$ do $\mathrm{N}$ ingerido $\mathrm{e}$ $46,84 \%$ do $\mathrm{N}$ absorvido (Tabela 3). Resultados semelhantes foram relatados por Henrique et al. (2003), que observaram valores médios de $\mathrm{N}$ ingerido (g/dia) e de $\mathrm{N}$ retido (g/dia) de 27,36 e 9,81, respectivamente, em estudo de ovinos que recebiam dieta com relação volumoso:concentrado de 20:80\%, Chandramoni et al. (2000a), ao estudarem o efeito de diferentes de níveis de concentrado sobre o balanço de $\mathrm{N}$ em ovinos, encontraram na relação $\mathrm{V}: \mathrm{C}$ de 30:70\%, 21,9g/dia de $\mathrm{N}$ consumido, $13,5 \mathrm{~g} /$ dia de $\mathrm{N}$ absorvido e $3,04 \mathrm{~g} /$ dia de $\mathrm{N}$ retido, valores inferiores aos observados no presente estudo. Macedo Junior et al. (2009), ao trabalharem com ovelhas gestantes, submetidas ou não à restrição nutricional, verificaram aumento no consumo de nitrogênio para ovelhas com dois fetos e sem restrição nutricional. Os autores acreditam que esse aumento se deva à maior inclusão de grãos e na maior quantidade proteína (na dieta desses animais). O mesmo foi verificado por Macedo Junior et al. (2010), Benevides et al. (2011) e Macedo Junior et al. (2012).

Os diferentes tamanhos de partícula utilizados não afetaram o balanço de $\mathrm{N}$ $(\mathrm{P}>0,05)$. Embora pudesse ser esperado aumento na retenção de $\mathrm{N}$, com a redução do tamanho de partícula do volumoso e com a maior área de superfície exposta ao ataque microbiano, não se verificou um aumento no $\mathrm{N}$ absorvido. Segundo Firkins et al. (1986), a moagem da forragem diminui a extensão da digestão ruminal do alimento, aumenta a ingestão de MS e pode aumentar a eficiência de síntese de proteína microbiana. Esses autores também não verificaram efeito do tamanho de partícula do volumoso sobre a digestibilidade total de $\mathrm{N}$, em novilhos sujeitos a feno de gramínea picado, grosseira ou finamente, numa relação $\mathrm{V}: \mathrm{C}$ de 70:30\%. Isso pode ter ocorrido devido à passagem mais rápida das partículas menores através do trato gastrintestinal, o que ocasiona, consequentemente, efeito compensatório no intestino grosso (fermentação no ceco) Benevides et al. (2007). Também se deve destacar a baixa participação proporcional do volumoso na dieta do presente ensaio, além do pouco efeito da digestibilidade do $\mathrm{N}$ do volumoso sobre a digestibilidade total do $\mathrm{N}$.

É sabido que os principais fatores que influenciam a retenção de $\mathrm{N}$ no organismo do ruminante são os teores de $\mathrm{N}$ e a energia da dieta, fatores estes semelhantes nas dietas experimentais. Assim, permite-se dizer que, mesmo com alta quantidade de concentrado nas dietas, o balaço de nitrogênio foi positivo, de modo a proporcionar ganhos ao animal.

Para Saenz (2005), quanto mais rápido a digesta flui do rúmen, menos tempo os micro-organismos têm para fermentar a mesma, o que diminui, portanto, a digestibilidade. A taxa de passagem afeta a degradação microbiana e, consequentemente, o crescimento microbiano. O tamanho da população microbiana também será reduzido devido à passagem mais rápida. Por causa do tempo de residência mais curto, a exigência de energia de mantença dos micro-organismos ruminais é reduzida e, portanto, mais biomassa microbiana pode ser produzida por unidade de energia gerada pelo substrato (BOUDON \& PEYRAUD, 2001).

Como observado no ensaio de consumo e digestibilidade, a frequência de alimentação não influenciou $(\mathrm{P}>0,05) \mathrm{o}$ consumo e a digestibilidade da matéria seca (MS) e do N, o que explica os resultados observados para o balanço de $\mathrm{N}$ terem sido semelhantes. $\mathrm{O}$ aumento na frequência de alimentação pode 
Rev. Bras. Saúde Prod. Anim., Salvador, v.13, n.3, p.732-744 jul./set., 2012 http://www.rbspa.ufba.br ISSN 15199940

melhorar a ingestão de MS e a conversão alimentar, principalmente, por garantir ambiente ruminal mais estável e adequado para o crescimento e ação da microbiota ruminal (DeVRIES et al., 2005). Isso também poderia garantir maior digestibilidade dos nutrientes, inclusive a de $\mathrm{N}$.

Porém, no presente trabalho esses efeitos não foram observados. Deve-se ressaltar que tanto os animais que recebiam a dieta na frequência de duas vezes ao dia (F2), como os que recebiam quatro vezes ao dia (F4), tiveram livre acesso ao alimento fresco durante todo o dia. Além disso, não existiu competição entre os animais, devido à forma de alojamento, o que pode ter garantido consumo mais constante ao longo do dia e explicar essa ausência de efeitos para o aumento da frequência de alimentação.
Os tratamentos $(\mathrm{F} 2$ e $\mathrm{F} 4$ e $2 \mathrm{~mm}$, $5 \mathrm{~mm}, 10 \mathrm{~mm}$ e $25 \mathrm{~mm}$ ) e as interações entre eles não afetaram o metabolismo energético dos ovinos ( $\mathrm{P}>0,05)$, conforme apresentado nas Tabelas 4 e 5 . Esse resultado permite dizer que os animais utilizados no experimento e nessas condições conseguiram manter a homeostase ruminal, uma vez que não houve presença de quaisquer distúrbios nutricional.

A produção de $\mathrm{CH}_{4}$ variou de 1,00 a $1,72 \mathrm{~L} / \mathrm{UTM} /$ dia e apresentou valor médio de $1,45 \mathrm{~L} / \mathrm{UTM} /$ dia que foi superior ao observado por Chandramoni et al. (2000a) (0,87L/UTM/dia) na relação V:C $30: 70 \%$. Blaxter \& Clapperton (1965), ao revisar a literatura, encontraram produção média de 29,9L/dia, valor próximo ao observado no presente trabalho (28,0L/dia).

Tabela 4. Médias e coeficientes de variação (CV) dos parâmetros da respirometria nos diferentes tratamentos

\begin{tabular}{|c|c|c|c|c|c|c|c|c|c|c|}
\hline \multirow{3}{*}{ Item } & \multicolumn{8}{|c|}{ Tratamentos } & \multirow{3}{*}{ Média } & \multirow{3}{*}{$\begin{array}{l}\mathrm{CV} \\
(\%)\end{array}$} \\
\hline & \multicolumn{4}{|c|}{$\mathrm{F} 2$} & \multicolumn{4}{|c|}{$\mathrm{F} 4$} & & \\
\hline & $2 \mathrm{~mm}$ & $5 \mathrm{~mm}$ & $10 \mathrm{~mm}$ & $25 \mathrm{~mm}$ & $2 \mathrm{~mm}$ & $5 \mathrm{~mm}$ & $10 \mathrm{~mm}$ & $25 \mathrm{~mm}$ & & \\
\hline UTM & 17,90 & 18,40 & 20,50 & 20,10 & 18,90 & 21,40 & 18,80 & 18,60 & 19,32 & - \\
\hline $\mathrm{CONSO}_{2} *$ & 20,72 & 25,76 & 20,85 & 24,72 & 22,91 & 25,11 & 23,66 & 23,73 & 23,43 & 19,07 \\
\hline $\mathrm{PCO}_{2} *$ & 21,67 & 25,85 & 21,40 & 24,68 & 24,75 & 25,84 & 24,67 & 26,19 & 24,38 & 19,14 \\
\hline $\mathrm{RQ}^{*}$ & 1,04 & 1,01 & 1,03 & 1,00 & 1,10 & 1,05 & 1,06 & 1,11 & 1,04 & 6,05 \\
\hline $\mathrm{PCH}_{4} *$ & 1,21 & 1,59 & 1,00 & 1,44 & 1,53 & 1,72 & 1,55 & 1,69 & 1,45 & 40,91 \\
\hline NUR* & 13,16 & 15,10 & 12,77 & 13,37 & 15,10 & 13,90 & 13,34 & 12,95 & 13,70 & 23,46 \\
\hline $\mathrm{PC}^{*}$ & 102,6 & 127,1 & 100,9 & 123,2 & 112,3 & 119,1 & 116,2 & 120,4 & 115,09 & 18,58 \\
\hline
\end{tabular}


Tabela 5. Médias e coeficientes de variação (CV) dos parâmetros do metabolismo energético nos diferentes tratamentos

\begin{tabular}{|c|c|c|c|c|c|c|c|c|c|c|}
\hline \multirow{3}{*}{ Item } & \multicolumn{8}{|c|}{ Tratamentos } & \multirow{3}{*}{ Média } & \multirow{3}{*}{$\begin{array}{l}\mathrm{CV} \\
(\%)\end{array}$} \\
\hline & \multicolumn{4}{|c|}{$\mathrm{F} 2$} & \multicolumn{4}{|c|}{ F4 } & & \\
\hline & $2 \mathrm{~mm}$ & $5 \mathrm{~mm}$ & $10 \mathrm{~mm}$ & $25 \mathrm{~mm}$ & $2 \mathrm{~mm}$ & $5 \mathrm{~mm}$ & $10 \mathrm{~mm}$ & $25 \mathrm{~mm}$ & & \\
\hline EBI* & $4.700,5$ & $5.922,1$ & $4.449,7$ & $5.409,6$ & $6.365,6$ & $5.999,6$ & $5.897,2$ & $5.320,5$ & $5.508,1$ & 23,80 \\
\hline EBFEZ* & $1.030,8$ & $1.182,3$ & 778,0 & 941,7 & $1.059,4$ & $1.062,3$ & $1.209,5$ & 985,0 & $1.031,1$ & 21,30 \\
\hline ED* & $3.669,7$ & $4.739,8$ & $3.671,7$ & $4.467,9$ & $5.306,2$ & $4.937,3$ & $4.687,7$ & $4.335,5$ & $4.476,9$ & 23,40 \\
\hline EBUR* & 144,36 & 255,49 & 148,87 & 142,23 & 181,62 & 156,99 & 160,89 & 215,00 & 175,68 & 63,24 \\
\hline $\mathrm{ECH}_{4} *$ & 203,90 & 275,44 & 193,62 & 273,62 & 272,42 & 346,32 & 275,08 & 295,4 & 266,97 & 42,27 \\
\hline $\mathrm{EM}^{*}$ & 3321,4 & 4208,8 & 3329,2 & 4052,0 & 4852,2 & 4433,9 & 4251,7 & 3825,2 & 4034,3 & 23,32 \\
\hline $\mathrm{ECH}_{4} / \mathrm{EB}^{*}$ & 4,34 & 4,65 & 4,35 & 5,06 & 4,28 & 5,77 & 4,66 & 5,55 & 4,83 & 36,20 \\
\hline $\mathrm{ECH}_{4} / \mathrm{ED}^{*}$ & 5,56 & 5,81 & 5,27 & 6,12 & 5,13 & 7,01 & 5,87 & 6,81 & 5,94 & 37,50 \\
\hline $\mathrm{PC}^{*}$ & $1.836,3$ & $2.338,1$ & $2.068,6$ & $2.476,0$ & $2.122,5$ & $2.549,8$ & $2.184,4$ & $2.239,9$ & $2.226,9$ & 21,36 \\
\hline EL* & $1.485,1$ & $1.870,7$ & $1.260,6$ & $1.576,0$ & $2.729,7$ & $1.884,1$ & $2.067,3$ & $1.585,2$ & $1.807,3$ & 62,2 \\
\hline
\end{tabular}

*Não houve diferença entre tratamentos. F2 - frequência de alimentação duas vezes ao dia; F4 - frequência de alimentação quatro vezes ao dia; TP2 - tamanho de partícula de $2 \mathrm{~mm}$; TP5 - tamanho de partícula de $5 \mathrm{~mm}$; TP10 - tamanho de partícula de $10 \mathrm{~mm}$; TP25 - tamanho de partícula de $25 \mathrm{~mm}$. EBI - energia bruta ingerida (kcal/dia); EBFEZ - energia bruta das fezes (kcal/dia); ED - energia digestível (kcal/dia); EBUR - energia bruta da urina (kcal/dia); ECH ${ }_{4}$ - energia do metano (kcal/dia); EM - energia metabolizável (kcal/dia); $\mathrm{ECH}_{4} / \mathrm{EB}$ - energia do metano como porcentagem da $\mathrm{EB} ; \mathrm{ECH}_{4} / \mathrm{ED}$ - energia do metano como porcentagem da ED; $\mathrm{PC}$ - produção de calor (kcal/dia); EL - energia líquida (kcal/dia). 
De acordo com Blaxter \& Clapperton (1965), os principais fatores que afetam a produção de $\mathrm{CH}_{4}$ são a fonte de volumoso, o tipo de carboidrato da dieta e a taxa de fermentação do mesmo, fatores semelhantes nas dietas experimentais, o que pode ter acarretado a igualdade de resultados encontrados. Segundo Van Soest (1994), a oferta de dieta com elevada proporção de carboidratos solúveis resulta em ambiente ruminal inadequado para o crescimento das bactérias metanogênicas. Esse ambiente é caracterizado pelo abaixamento do $\mathrm{pH}$, o que prejudica certas populações de protozoários e de bactérias metanogênicas, de modo a reduzir a produção de $\mathrm{CH}_{4}$.

A PC inclui o calor produzido como resultado dos processos fermentantivos do trato gastrintestinal e o liberado no metabolismo intermediário e representa em média 25,0 a 40,0\% da EB consumida (NRC, 2007). Esta apresentou valor médio de $115,0 \mathrm{kcal} / \mathrm{UTM} / \mathrm{dia}$, enquanto Chandramoni et al. (2000a) verificaram produção média de $92,0 \mathrm{kcal} / \mathrm{UTM} / \mathrm{dia}$. Tais diferenças podem estar associadas a fatores ambientais e biológicos (sexo, idade, raça, ganho), que influenciam, diretamente, a taxa metabólica dos animais. Entre os fatores ambientais destaca-se a restrição alimentar, visto que animais em jejum reduzem expressivamente a produção de calor, como relatado por Chandramoni et al. (2000b). Tais autores verificaram PC média de 53,2kcal/UTM/dia em ovinos que recebiam dieta com uma relação V:C 30:70\% para atender às necessidades de mantença.

$\mathrm{O}$ consumo médio de EB foi de $5.508,10 \mathrm{kcal} / \mathrm{dia}$ e variou de $4.449,70 \mathrm{a}$ $6.365,60 \mathrm{kcal} / \mathrm{dia}$. Chandramoni et al. (2000a) verificaram consumo médio de EB de 3.840,0kcal/dia na relação V:C de $30: 70 \%$, valor inferior à média observada. Santos et al. (2009), ao trabalharem com ovinos sujeitos a diferentes fontes de canola, não verificaram diferença no consumo de energia e proteína. Verificou-se consumo médio de ED de $4.476,97 \mathrm{kcal} / \mathrm{dia}$, valor superior ao observado por Chandramoni et al. (2000a), de 2.310,0kcal/dia. A eficiência de uso da EB como ED foi de $81,27 \%$, valor bem superior aos $60,0 \%$ observados por Chandramoni et al. (2000a) e próximo ao sugerido pelo NRC (2007) de 70,0 a 80,0\%, para dietas com elevada proporção de concentrado. Portanto, essa elevada eficiência ocorreu, provavelmente, devido à alta inclusão de concentrado na dieta o que afetou positivamente a digestão dos nutrientes (ALVES et al., 2003; CABRAL, et al., 2008).

A energia perdida na urina representa, em média, 4 a 5\% da EB (NRC, 2007), valor superior ao observado, de 3,19\% (175,68kcal/dia). Segundo o NRC (2007), os principais fatores que influenciam a EBUR são as concentrações dietéticas de proteína e gordura e a relação $\mathrm{V}: \mathrm{C}$, fatores estes semelhantes nas diferentes dietas experimentais.

De acordo com o NRC (2007), a energia perdida através dos gases da digestão podem representar de 5-8\% da EB consumida. A energia liberada na forma de $\mathrm{CH}_{4}$ variou de 193,62 a $346,32 \mathrm{kcal} / \mathrm{dia}$ e foi, em média, de $266,70 \mathrm{kcal} / \mathrm{dia}$, valor superior aos $111,0 \mathrm{kcal} / \mathrm{dia}$ observada por Chandramoni et al. (2000a). As perdas de energia pelo $\mathrm{CH}_{4}$, expressas como porcentagem da EB e da ED foram, respectivamente, de 4,88 e 5,94\%, números superiores aos relatados por Chandramoni et al. (2000a) de 2,98 e $4,87 \%$, para dietas com elevada proporção de concentrado. Fatores como a relação $\mathrm{V}: \mathrm{C}$, assim como a qualidade e o processamento dos alimentos podem afetar a produção e, consequentemente, a energia perdida na forma de metano. Além disso, como 
relatado anteriormente, dietas como as do presente experimento, com elevada proporção de concentrado $(75 \%)$ podem provocar mudanças no padrão de fermentação ruminal, de modo a causar prejuízos à população de bactérias metanogênicas e favorecer a produção de propionato, o que resulta em redução na produção de $\mathrm{CH}_{4}$, com aumento na eficiência energética.

A EM variou de $3.321,4 \mathrm{kcal} / \mathrm{dia}$ a $4.852,2 \mathrm{kcal} / \mathrm{dia}$, com valor médio de $4.034,32 \mathrm{kcal} / \mathrm{dia}$, sendo $73,24 \%$ da EBI. Esse valor encontra-se abaixo do fator de conversão de 0,82 ( $82 \%$ da ED) adotado pelo NRC (2007). A EL disponível para as atividades de mantença e produção, apresentou valor médio $1.807,35 \mathrm{kcal} /$ dia e foi superior ao obtido por Chandramoni et al. (2000a), de 717,0 kcal/dia com dieta composta de $70 \%$ de concentrado. Segundo o NRC (2007) é essa energia que o animal irá utilizar para a atividade muscular, no reparo e na proliferação tecidual e em processos involuntários. A EL também será utilizada nos processos produtivos como crescimento, lactação, reprodução e produção de lã.

O tamanho de partícula do volumoso e a frequência de alimentação, em dietas com elevada proporção de concentrado, não tem efeito sobre $\mathrm{o}$ balanço de nitrogênio e o metabolismo energético em ovinos. Os animais conseguiram manter a homeostase ruminal, mesmo sobre condições de desafio.

\section{REFERÊNCIAS}

ALVES, K.S.; CARVALHO, F.F.R.; VERAS, A.S.C.; FERREIRA, M.A.; COSTA, R.G.; SANTOS, E.P.; FREITAS, C.R.G.; SANTOS JÚNIOR, C.M.; ANDRADE, D.K.B. Níveis de energia em dietas para ovinos Santa
Inês: Digestibilidade Aparente. Revista

Brasileira de Zootecnia, v.32, n.6, p.1962-1968, 2003.

ALVES, K.S; CARVALHO, F.F.R.; VÉRAS, A.S.C.; ANDRADE, M.F.; COSTA, R.G.; BATISTA, A.M.V.; MEDEIROS, A.N.; SOUTO MAIOR JÚNIOR, R.J.; ANDRADE, D.K.B. Níveis de energia em dietas para ovinos Santa Inês: desempenho. Revista Brasileira de Zootecnia, v.32, n.6, p.1937-1944, 2003. Supl.2.

ARRUDA, D.S.R.; CALIXTO JUNIOR, M.; JOBIM, C.C.; SANTOS, G.T. Efeito de diferentes volumosos sobre os constituintes sanguíneos de vacas da raça holandesa. Revista Brasileira de Saúde e Produção Animal [Online], v.9, n.1, p.35-44, 2008.

AMERICAN SOCIETY DA AGRICULTURA ENGINEERS ASAE. Standards S424. Method of determining and expressing particle size of chopped forage materials by sieving. Eng. St. Joseph, MI, 2001.

BENEVIDES, Y.I.; CÂNDIDO, J.D.; NEIVA, J.N.M.; BORGES, I.; SILVA, A.G.M.; SILVA, R.G. Composição e degradabilidade da dieta de ovinos em capim Tanzânia com três períodos de descanso. Archivos de Zootecnia, v.56, n.214, p.215-226, 2007.

BENEVIDES, Y.I.; CAMPOS, W.E.; RODRIGUEZ, N.M.; MACEDO JUNIOR, G.L.; BORGES, I.; FERREIRA, M.I.C.; BRITO, T.S.; PIRES, C.P. Comportamento ingestivo de ovelhas submetidas ou não à restrição nutricional durante a gestação. Archivos de Zootecnia, v.60, n.232,p.891-901, 2011. 
Rev. Bras. Saúde Prod. Anim., Salvador, v.13, n.3, p.732-744 jul./set., 2012 http://www.rbspa.ufba.br ISSN 15199940

BLAXTER, K.L.; CLAPPERTON, J.L. Prediction of the amount of methane produced by ruminants. Britsh Journal Nutrititon, v.19, p.511-522, 1965.

BLAXTER, K.L. The nutritive value of feeds as sources of energy: a review. Journal Dairy Science, v.39, p.1396$1424,1956$.

BOUDON, A.; PEYRAUD, J.L. The release of intracellular constituents from fresh ryegrass (Lolium perenne L.) during ingestive mastication in dairy cows: effect of intracellular constituent, season and stage of maturity. Animal Feed Science and Technology, v. 93, n.3-4, p.229-245, 2001.

BROUWER, E. Report of sub-committee on constants and factors. In:

SYMPOSIUM ON ENERGY METABOLISM, 3., 1965, London. Proceedings... London: Academic Press, 1965.

CABRAL, L.; SANTOS, J.W.; ZERVOUDAKIS, L.T.; ABREU, J.G.; SOUZA, A.L.; RODRIGUES, R.C. Consumo e eficiência alimentar em cordeiros confinados. Revista Brasileira de Saúde e Produção Animal [Online], v.9, n.4, p.703-714, 2008.

CHANDRAMONI; C.M. TIWARI; JADHAO, S.B.; KHAN, M.Y. Energy metabolism with particular reference to methane production in Muzaffarnagari sheep fed rations varying in roughage to concentrate ratio. Animal Feed Science Technologique, v.83, p.287300, 2000a.

CHANDRAMONI; C.M. TIWARI; JADHAO, S.B.; KHAN, M.Y. Fast heat production of Muzaffarnagari sheep. Small Ruminant Research, v.36, p.43-47, 2000 b.
DeVRIES, T.J.; VON

KEYSERLINGK, M.A.G.;

BEAUCHEMIN, K.A. Frequency of feed delivery affects the behavior of lactating dairy cows. Journal Dairy Science, v.88, p.3533-3562, 2005.

DOREAU, B.; DOREAU, M. Influence of drastic underfeeding on ruminal digestion in sheep. Animal Research, v.50, n.6, p.451-462, 2001.

DOREAU, M.; MICHALETDOREAU, B.; GRIMAUD, P.; ATTI, N.; NOZIÈRE, P. Consequences of underfeeding on digestion and absorption in sheep Small Ruminant Research, v.49, p.289-301, 2003.

FIRKINS, J.L.; BERGER, L.L; MERCHEN, N.R.; FAHEY JUNIOR, G.C. Effects of forage particle size, level of feed intakes and supplemental protein degradability on microbial protein synthesis and site of nutrient digestion in steers. Journal Animal Science, v.62, p.1081-1094, 1986.

FURUSHO-GARCIA, I.F.; PEREZ, J.R.O.; OLIVEIRA, M.V.M.

Componentes corporais e órgãos internos de cordeiros Texel $\mathrm{x}$ Bergamácia, Texel x Santa Inês e Santa Inês puros, terminados em confinamento, com casca de café como parte da dieta. Revista Brasileira de Zootecnia, v.32, n.6, p.1992-1998, 2003.

FRANÇA, S.R.L.; GONZAGA NETO, S.; PIMENTA FILHO, E.C.; MEDEIROS, A.N.; TORREÃO, J.N.C.; MARIZ, T.M.A.; COSTA, R.G.

Comportamento ingestivo de ovelhas Morada Nova no terço final de gestação com níveis de energia metabolizável na dieta. Revista Brasileira de Saúde e Produção Animal [Online], v.10, n.1, p.73-84, 2009. 
Rev. Bras. Saúde Prod. Anim., Salvador, v.13, n.3, p.732-744 jul./set., 2012 http://www.rbspa.ufba.br ISSN 15199940

GOMES, S.P.; BORGES, A.L.C.C.; BORGES, I.; MACEDO JUNIOR, G.L.; SILVA, A.G.; PANCOTI, C.G. Efeito do tamanho de partícula do volumoso e da frequência de alimentação sobre o consumo e a digestibilidade em ovinos. Revista Brasileira de Saúde e Produção Animal [Online], v.13, n.1, p.137-149, 2012.

HENRIQUE W.; SAMPAIO, A.A.M.; LEME, P.R.; ALLEONI, G.F.; LANNA, D.P.D.; MALHEIROS, E.B.

Digestibilidade e balanço de nitrogênio em ovinos alimentados à base de dietas com elevado teor de concentrado e níveis crescentes de polpa cítrica peletizada. Revista Brasileira de Zootecnia, v.32, n.6, p.2007-2015, 2003. Supl. 2.

KONONOFF, P.J.; HEINRICHS, A.J. The effect of corn silage particle size and cottonseed hulls on cows in early lactation. Journal of Dairy Science, v.86, p.2438-2451, 2003.

LAMMERS, B.P.; BUCKMASTER, D.R.; HEINRICHS, A.J. A simple method for the analysis of particle sizes of forage and total mixed rations. Journal of Dairy Science, v.79, p.922-928, 1996.

MACEDO JÚNIOR, G.L.; PÉREZ, J.R.O.; ALMEIDA, T.R.V. Influência de diferentes níveis de FDN dietético no consumo e digestibilidade aparente de ovelhas Santa Inês. Ciência e

Agrotecnologia, v.30, p.547-553, 2006.

MACEDO JUNIOR, G.L.; PEREZ, J.R.O.; DE PAULA, O.J.; ALMEIDA, T.R.V.; ASSIS, R.M.; FRANÇA, P.M.; SILVA, V.B.; BORGES, I.; BAIÃO, A.A.F. Níveis de fibra em detergente neutro na alimentação de ovelhas Santa Inês gestantes. Arquivo Brasileiro de Medicina Veterinária e Zootecnia, v.61, n.1, p.196-202, 2009.
MACEDO JÚNIOR, G.L.; FERREIRA, M.I.C.; BORGES, I.; SILVA, V.B.; COUTO, J.R.L.; CAVALCANTI, L.F.L. Consumo e digestibilidade aparente das frações fibrosas por ovelhas gestantes submetidas ou não à restrição nutricional. Revista Brasileira de Saúde e Produção Animal [Online], v.11, n.1, p.179-192, 2010

MACEDO JUNIOR, G.L.; SOUSA, L.F.; GODOI, F.N.; PEREZ, J.R.O.; FRANÇA, P.M.; ALMEIDA, T.R.V.; PAULA, O.J.; ASSIS, R.M. Consumo, digestibilidade aparente e balanço de nitrogênio em ovelhas alimentadas com diferentes níveis de fibra em detergente neutro. Ciência Animal Brasileira, v.13, n.1, p.33-40, 2012.

NATIONAL RESEARCH COUNCIL NRC. Nutrient requirements of sheep. 6.ed. Washington, D.C.: National Academic Press, 1985. 99p.

NATIONAL RESEARCH COUNCIL NRC. Nutrient requirements of small ruminants: sheep, goats, cervids, and new world camelids. Washington, D.C.: National Academic Press, 2007. $362 p$.

RODRIGUEZ, N.M.; CAMPOS, W.E; LACHICA, M.L.; BORGES, I.; GONÇALVES, L.C.; BORGES, A.L.C.C.; SALIBA, E.O.S. A calorimetry system for metabolism trials. Arquivo Brasileiro Medicina Veterinária e Zootecnia, v.59, n.2, p.495-500, 2007.

SAENZ, E.A.C. Modelagem da redução do tamanho de partículas na alimentação de ruminantes. Ciência e Agrotecnologia, v.29, n.4, p.886-893, 2005. 
Rev. Bras. Saúde Prod. Anim., Salvador, v.13, n.3, p.732-744 jul./set., 2012 http://www.rbspa.ufba.br ISSN 15199940

SANTOS, V.C.; EZEQUIEL, J.M.B.; OLIVEIRA, P.S.N.; GALATI, R.L.; BARBOSA, J.C. Consumo e digestibilidade em ovinos alimentados com grãos e subprodutos da canola. Revista Brasileira de Saúde Produção Animal [Online], v.10, n.1, p.96-105, 2009.

SILVA, D.J.; QUEIROZ, A.C. Análise de alimentos: métodos químicos e biológicos. Viçosa: Universidade Federal de Viçosa, 2002. 235p.

SILVA, H.G.O.; PIRES, J.A.V.; SILVA, F.F. Farelo de Cacau (Theobroma cacao L.) e Torta de Dendê (Elaeis guineensis, Jacq) naAlimentação de Cabras em Lactação: Consumo e Produção de Leite. Revista Brasileira de Zootecnia, v.34, n.5, p.1786-1794, 2005.

SILVA, H.G.O.; PIRES, J.A.V.; CUNHA, P.A.; Digestibilidade de dietas contendo silagem de capimelefante amonizado e farelo de cacau ou torta de dendê em ovinos Revista Brasileira de Zootecnia, v.36, n.2, p.499-506, 2007
VAN SOEST, P.J. Nutritional ecology of the ruminant. 2.ed. Ithaca: Cornell University Press, 1994, 476p.

VAN SOEST, P.J.; ROBERTSON, J.B.; LEWIS, B.A. Methods for dietary fiber, neutral detergent fiber, and nonstarch polysaccharides in relation to animal nutrition. Journal Dairy Science, v.74, p.3583-3597, 1991.

ZANINE, A.; MACEDO JÚNIOR, G. Importância do consumo da fibra para nutrição de ruminantes. Revista Eletrônica de Veterinária REDVET, v.7, n.2, 2006 Disponível em: <http://www.veterinaria.org/revistas/red vet/n020207/020718.pdf > . Acesso em: 20 jan. 2011.

Data de recebimento: 06/09/2011

Data de aprovação: 08/05/2012 